\section{Visual and auditory processing of common pattern class structure*}

\author{
LEONA S. AIKEN $\dagger$ †† and LAWRENCE R. GRIFFIN \\ Temple University, Philadelphia, Pennsylvania 19122
}

Visual and auditory classification of equivalent class-structured patterns were examined. Underlying patterns from two classes were translated into auditory tone sequences and visual polygons. All Ss classified 50 visual patterns and their direct auditory analogs. Visual classification accuracy exceeded auditory accuracy $(p<.01)$; however, auditory accuracy improved when auditory classification was preceded by the visual task $(p<.01)$. Based on group data, classification strategies appeared similar across modalities, with accuracy of classification of individual patterns predicted to the same degree by common measures of physical class structure across modalities. Ss' drawings of the prototypes also suggested a common strategy across modalities. While group data suggest some consistency of classification strategy across modalities, individual Ss were not at all consistent in their visual and auditory classifications.

The processes of pattern perception, e.g., extraction of distinctive features and prototypic characteristics, are often conceptualized as being modality independent, as Gibson (1962) has argued for vision and touch. In discussions of pattern processing, the human observer is often described as an "intuitive statistician" who extracts lawful structure from patterned input, regardless of the source of this input (Peterson \& Beach, 1967). It has repeatedly been suggested (e.g., Brown \& Owen, 1967; Garner, 1962) that the relevant characteristics of patterned stimulation are the lawful distributional characteristics of this stimulation which occur over sets of events, without reference to the modality or format of the stimulus input.

It is possible to manipulate the structure inherent in a collection of patterns through the choice of the rules by which the patterns are generated. Imposition of structure might be accomplished by constraining patterns to form clusters or classes, through the manipulation of the values of pattern elements or interelement transitions. Evans and Mueller (1966) and Aiken and Brown (1971) have described similar methods for generating class-structured patterns.

Once a class-structured set of patterns is constructed, it is possible to translate the underlying patterns into a

\footnotetext{
*This research was supported by Temple University Grant-In-Aid 400-101-89.

tRequests for reprints should be sent to the Department of Psychology, Temple University, Philadelphia, Pa. 19122.

t+The authors express their appreciation to Clifford Taylor, Professor of Music, Temple University, for his preparation of the auditory patterns and to Dr. D. R. Brown, Dr. S. H. Evans, and particularly to Dr. I. Pollack for their helpful comments concerning the manuscript.
}

variety of formats suitable for presentation to the various modalities. If it is the case that we respond to pattern structure independent of format or input modality, then observers should be able to extract the underlying structure regardless of the input properties of the stimulation.

Repeated tests have been made of the ability of children and adults to extract the structure inherent in classes of visual patterns (e.g., Aiken, 1972; Aiken \& Brown, 1971; Aiken \& Williams, 1972; Bersted, Brown, \& Evans, 1969; Evans \& Arnoult, 1967). The result that observers are sensitive to class-defining attributes of visual patterns is limited by neither pattern format, e.g., histoform vs polygon, nor by specific classification task employed, e.g., serial classification of individual patterns, same-different judgments, oddity judgments. Physical measures of the extent to which individual patterns adhere to the class generation rules have been found predictive of the accuracy of classification of both groups of patterns and individual patterns.

It is of interest to ask whether such class rule sensitivity is a property of visual pattern processing or of a more general example of modality-independent sensitivity to lawful pattern structure. Based on previous comparisons of visual and auditory processing of relatively simple binary patterns (Garner \& Gottwald, 1968; Rubenstein \& Gruenberg, 1971), it appears that the latter alternative of modality-independent pattern structuring is more likely the case.

The present study was designed as a test of the modality independence of pattern processing, and specifically examined pattern classification by the same observers across the visual and auditory modalities. A pattern generation procedure for constructing equivalent visual and auditory pattern classes was employed. In contrast with the relatively simple binary patterns employed in earlier comparisons of visual and auditory pattern processing, the patterns used herein were more complex in that each pattern element could take on a variety of values. Measures of adherence of individual patterns to the generation rule were applied to the accuracy of classification of individual patterns in both modalities. Lastly, performance levels of individual Ss were compared across modalities.

\section{Pattern Generation, Translation, and Measurement}

Generation. The Vargus 9 procedure of Evans (Evans \& Mueller, 1966) was employed to construct two classes of patterns suitable for translation to visual and auditory representations. Each pattern generated by this procedure is actually a sequence of numerical values. Each numerical value of the sequence is mapped into a pattern element, the ordered series of which constitutes the pattern.

To generate two classes of patterns, two prototypic sequences of numbers, each of length eight, were randomly chosen with $\left(3 \leqslant p_{i k} \leqslant 7\right)$, where $p_{i k}$ is the value of the $i^{\text {th }}$ element of Prototype k (see Fig. 1). Variants of the prototype were then constructed by the addition to each element of a random value $r_{i j k}$, where $\left(-2 \leqslant r_{i j k} \leqslant 2\right)$ for the ith element of Variant $\mathrm{j}$ of Prototype $\mathrm{k}$. In order that the patterns generated from a single prototype would resemble the prototype and would form a statistical cluster in terms of element values, the probabilities that the prototypic valyes would be changed by $\pm 2, \pm 1$, or 0 units were constrained. The probability of change by \pm 2 units was .06 ; by \pm 1 unit, .11; and by 0 units, .66. Constraining the probabilities in this way reduces the uncertainty of each column height from the $\log _{2} 5=$ 2.322 bits of uncertainty which would be obtained if the probability of all values of change were equal. For the specific probabilities employed, the uncertainty was reduced by half, yielding a constraint redundancy (Evans, 1967 ) of $R_{c}=50 \%$.

Translation. All numerical sequences were translated into visual and auditory representations (see Fig. 1). Eight-note auditory sequences were created on a Moog Synthesizer III by mapping the numerical value of each element into a pure tone of 265-412 Hz. Tones employed were separated according to an even-tempered scale. Each note was 0.6 sec in duration in accord with the high level of accuracy of learning of 


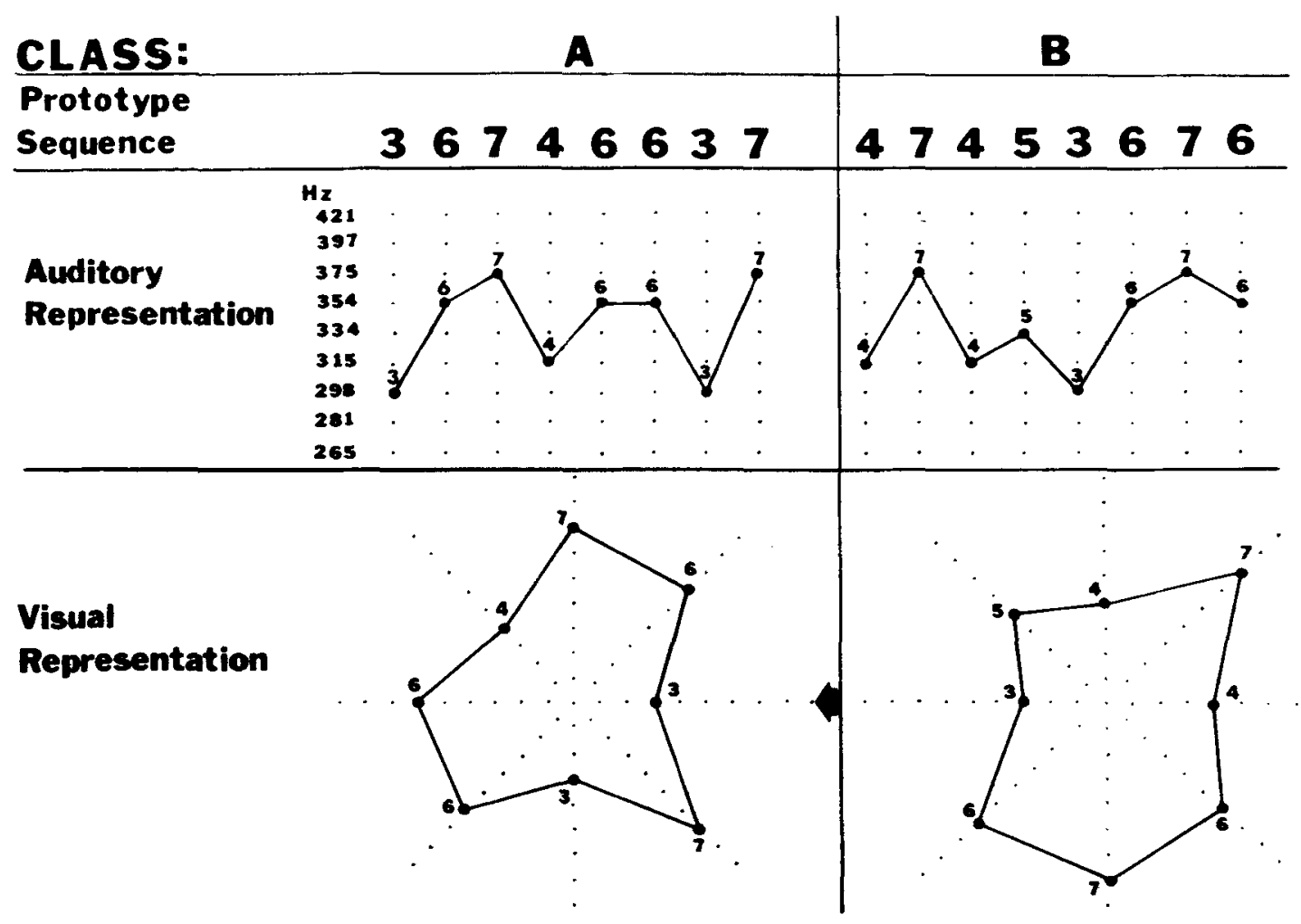

Fig. 1. Procedure for translation of underlying numerical sequences to auditory and visual patterns, shown for prototypes used in the experiment.

auditory sequences in the range of this presentation rate found by Garner and Gottwald (1968). Visual patterns were created by mapping the numerical value of each pattern element into the length of a radial of an eight-sided polygon, with the first element mapped to the radial of $0 \mathrm{deg}$ rotation.

Measurement. It is to be noted that while all patterns were generated at a $50 \%$ constraint redundancy level, this overall constraint specifies structure in the set of patterns. Since the degree of perturbation of any individual column height is only determined probabilistically over the set of patterns, individual patterns generated at any single constraint level may vary widely in the extent to which they adhere to their own and the other prototype. Two measures which assessed the physical similarity of each pattern to both prototypes were thus calculated for each pattern. The first measure was the Pythagorean distance, DP $P_{\text {own }}$, of a pattern from its own prototype, calculated as

$$
\left[\sum_{i=1}^{8}\left(X_{i}-X_{p_{i}}\right)^{2}\right]^{1 / 2},
$$

where $X_{i}$ is the numerical value of the $i^{\text {th }}$ element of a pattern and $X_{p_{i}}$ is the numerical value of the corresponding element of the prototype. The second measure, DPother, was the Pythagorean distance of each pattern from the opposing prototype. These two measures calculated for a single pattern summarize the extent to which each pattern adheres to the class structure of its own vs the opposing class. Since each numerical sequence on which DP $P_{\text {own }}$ and DP other were calculated had been translated into a visual and an auditory representation, these measures assessed class rule adherence equivalently in both modalities. These measures, in sum, operationalize the physical structure of the pattern set in a modality-independent fashion. It would be expected that pattern classification accuracy should be a negative function of $D P_{\text {own }}$, i.e., the smaller the distance between a pattern and its prototype, the greater its accuracy of classification. The converse would be expected for DP other.

\section{Subjects}

The Ss were 40 undergraduate volunteers enrolled in psychology and education courses who were paid $\$ 2$ each for their participation.

\section{Procedure}

The Ss were assigned randomly to two groups of 20 each. Group A1-V2 received an auditory classification task followed by a visual classification task involving the direct analogs of the auditory patterns. Group V1-A2 received the same tasks in reversed order.

The Ss viewed (heard) 10 patterns from a single pattern class and were informed that these were members of a common class, $A$. They then sequentially viewed (heard) 50 patterns, 25 from each class. After each pattern, they indicated that the pattern was a member either of Class A or of a different class, B, by manually depressing one of two response buttons. The same procedure of 10 training patterns followed by 50 test patterns was then repeated in the second modality. After performance in each modality, Ss sketched the Class A and Class B prototypes on grids identical to those shown in Fig. 1.

In both modalities, the 50 patterns were arranged into five 10-pattern blocks, each containing 5 patterns from each class. The blocks were arranged into five orders of a balanced Iatin square. Four of the 20 Ss within each group were assigned randomly to each of the five block orders to control for order effects. In the visual modality, the occurrence of each pattern was cued with a light. The pattern appeared 1 sec later and was projected for $6 \mathrm{sec}$. In the auditory modality, the occurrence of each 
pattern was cued with a $1,000-\mathrm{Hz}$ later and was played three times with an 0.6 -sec break between each repetition. Ss were permitted to respond in each modality either during or after pattern presentation.

\section{RESULTS}

Three types of analyses were made of pattern classifications. First, overall performance levels in the two experimental groups were compared across modalities. Second, the consistency of classification of individual patterns was examined across modalities, with psychophysical analyses based on the measures $D P_{\text {own }}$ and DP other made of pattern classification within each modality. Third, the consistency of performance levels of individual Ss was examined across modalities.

\section{Overall Performance}

Mean visual classification accuracy exceeded auditory classification accuracy for both first modality of presentation (visual scores from V1-A2 vs auditory scores from A1-V2) and second modality (visual scores from A1-V2 vs auditory scores from V1-A2), as shown in Table 1. Auditory performance was more accurate when Ss performed the visual task first (auditory scores from A1-V2 vs V1-A2), though visual performance did not increase markedly when visual classification was preceded by the auditory task (visual scores from A1-V2 vs V1-A2).

These results were verified in a 2 by 2 by 5 analysis of variance of Group (V1-A2, A1-V2) by Presentation (first modality, second modality) by Trial Blocks (Trials 1-10, .., 41-50). There was no difference between the two groups in overall performance $[F(1,38)=1.60, p>.10]$, no effect of trial blocks $(F<1)$, and no interaction of blocks with any other factor ( $p>.10$ in all cases). As indicated in Table 1, performance increased from first modality of presentation to second modality of presentation $[F(1,38)=10.81, p<.01]$. However, tone. The note sequence began $1 \mathrm{sec}$

Table 1

Mean Number of Correct Classifications of Each Experimental Group in Each Modality

\begin{tabular}{ccc}
\hline $\begin{array}{c}\text { Experimental } \\
\text { Group }\end{array}$ & $\begin{array}{c}\text { First } \\
\text { Modality }\end{array}$ & $\begin{array}{c}\text { Second } \\
\text { Modality }\end{array}$ \\
\hline & Visual & Auditory \\
V1-A2 & 40.95 & 42.00 \\
& $(5.14)$ & $(5.59)$ \\
& Auditory & Visual \\
A1-V2 & 36.55 & 44.55 \\
& $(5.82)$ & $(3.49)$ \\
\hline
\end{tabular}

Note-Maximum number correct $=50$. Stan. dand deviations are given in parentheses.

differential improvement was indicated by the significant Presentation by Group interaction $[F(1,38)=17.28, p<.01]$.

Within the visual modality, performance accuracy did not differ across presentation times $[F(1,38)=$ 2.32, $p>.10]$. However, as stated above, a marked gain was noted in auditory performance when it was preceded by the visual task $[F(1,38)=$ $9.98, \mathrm{p}<.01]$. This improvement was noted, even though the auditory accuracy level at first presentation exceeded chance $[t(19)=8.12$, $\mathbf{p}<.01]$. Visual classification accuracy exceeded auditory accuracy for both first presentation $[F(1,38)=$ $15.25, p<.01]$ and second presentation $[F(1,38)=4.74$, $\mathrm{p}<.05]$. In sum, visual performance exceeded auditory performance, though auditory accuracy increased markedly when preceded by the visual task.

\section{Consistency of Classification}

Accuracy of Individual Patterns

Consistency of classification of individual patterns was examined within and across modalities. The number of correct responses to each pattern with each modality and presentation was calculated. This yielded four scores for each pattern. From Group V1-A2, the following two scores were calculated: (1) Visual, , the number of correct visual classifications, and (2) Auditory A $_{2}$ the number of correct auditory

Table 2

Correlations Among Number of Correct Classifications of Individual Patterns in Each Modality and Presentation

\begin{tabular}{|c|c|c|c|c|}
\hline \multirow[b]{2}{*}{ Modality } & \multicolumn{2}{|c|}{ Visual } & \multicolumn{2}{|c|}{ Auditory } \\
\hline & Visual $_{1}$ & Visual $_{2}$ & Auditory $_{1}$ & Auditory $_{2}$ \\
\hline $\begin{array}{l}\text { Visual }_{1} \\
\text { Visual }_{2}\end{array}$ & - & $\frac{.69+}{-}$ & $\begin{array}{l}.20 \\
.40 w t\end{array}$ & $\begin{array}{l}.13 w \\
.30 *\end{array}$ \\
\hline $\begin{array}{l}\text { Auditory }_{1} \\
\text { Auditory }_{2}\end{array}$ & & & - & $.63 t$ \\
\hline
\end{tabular}

Note-The $w$ appended to a correlation indicates a within-S correlation across modalities. All other correlations are between-S correlations.

$*_{p} \leqslant .05,+p \leqslant .01$ classifications. From Group A1-V2, corresponding scores were calculated: (3) Auditory, , the number of correct a uditory classifications, and (4) Visual $_{2}$, the number of correct visual classifications. The correlations among these four measures are shown in Table 2. Classification accuracy was significantly correlated across groups within each modality. For Group A1-V2, classification accuracy was significantly correlated across modalities, although this was not the case for Group V1-A2.

If Ss were sensitive to variations in the adherence of individual patterns to the class generation rules, then this sensitivity should be reflected in the extent to which individual patterns were classified correctly. As previously explained, the measures $\mathrm{DP}_{\text {own }}$ and DP other reflect adherence of individual patterns to the generation rule. A pattern should be most easy to classify (high accuracy scores) if it is close to its own prototype (small value of $\mathrm{DP}_{\mathrm{own}}$ ) and far from the other prototype (large value of $\mathrm{DP}_{\text {other }}$ ). Thus, if $D P_{\text {own }}$ and $D P_{\text {other }}$ were predictive of the classification accuracy of individual patterns, this would indicate that Ss were sensitive to the structure inherent in the pattern set.

To test this notion, $\mathrm{DP}_{\text {own }}$ and DP other were used as predictors in four regression analyses, with the individual pattern accuracy scores, Visual $_{1}$, Visual $_{2}$, Auditory ${ }_{1}$, and Auditory $_{2}$, as the dependent variables. The results of these analyses are given in Table 3. In all four regression analyses, $\mathrm{DP}_{\text {ow } n}$ was a significant predictor of classification accuracy of individual patterns, with the sign of thy regression coefficient in the expected negative direction for all four analyses. The sign of the regression coefficient for $D P_{\text {other }}$ was also in the predicted positive direction in each equation. However, only in the auditory analyses did $D P_{\text {other }}$ contribute significantly to the prediction of classification accuracy. The level of prediction accuracy was equivalent across modalities, as indicated by the order of magnitude of the multiple correlations (see Table 3).

\section{Consistency of}

Individual Ss' Performance

The performance of individual $\mathrm{Ss}$ was compared across modalities. The number of correct responses within each modality for each $S$ was uncorrelated across Ss within each experimental group: for V1-A2, r(18) $=.06, \mathrm{p}>.10$; for $\mathrm{A1}-\mathrm{V} 2, \mathrm{r}(18)=$ $-.15, p>.10$. To further examine $\mathrm{Ss}^{\prime}$ performance within the two modalities, their prototype drawings were scored for accuracy of prototype 
Table 3

Prediction of Accuracy of Classification of Individual Patterns by Measures of Adherence to Prototy pe

\begin{tabular}{lccc}
\hline & \multicolumn{3}{c}{$\begin{array}{c}\text { Standardized Regres- } \\
\text { sion Coefficients }\end{array}$} \\
\cline { 2 - 3 } Criterion & DP own & DP other & rmult \\
\hline Visual $_{1}$ & $-.57 \dagger$ & .02 & $.57 \dagger$ \\
Visual $_{2}$ & $-.71 \dagger$ & .16 & $.68 \dagger$ \\
Auditory $_{1}$ & $-.62 \dagger$ & $.25 *$ & $.60 \dagger$ \\
Auditory $_{2}$ & $-.56 \dagger$ & $.52 \dagger$ & $.65 \dagger$ \\
\hline
\end{tabular}

$* p \leqslant .05,+p \leqslant .01$

reproduction with a directional time series correlation (Strahan, 1971). (The drawings of several Ss could not be scored due to their failure to follow sketching instructions. Missing data are indicated below by stating reduced degrees of freedom for individual correlations.) The time series correlation assesses the extent to which the numerical transitions between adjacent elements within one variable follow those transitions in a second variable. It is given by $(s-d) / n$, where $s$ is the number of transitions between adjacent elements which are in the same direction for both variables, $d$ is the number of opposing transitions, and $n$ is the total number of transitions. ${ }^{1}$ This measure was taken to reflect the level of accuracy of prototype extraction by Ss in each modality. Ss' accuracy of reproduction of each prototype was uncorrelated across modalities: for Prototype A, $r(31)=.23, p>.10$; for Prototype $B, r(37)=.16, p>.10$. Prototype $A$ reproduction accuracy was, however, correlated with classification accuracy within each modality, indicating the convergence of these two indices as measures of class concept attainment: for auditory classification, $\mathrm{r}(38)=.49, \mathrm{p}<.01$; for visual classification, $\mathrm{r}(32)=.51$, $\mathrm{p}<.01$.

Protocols of individual Ss were also examined for contingency between errors on individual patterns across modalities. For each $\mathbf{S}$, chi square was calculated between the correctness of classification of individual patterns in two modalities. For no $S$ did this chi square reach significance at the .05 level; only one of the 40 chi squares approached significance at the .10 level. In sum, while level of prototype reproduction was related to performance within a modality, no evidence was found for consistency of individual $S$ performance across modalities, in level of classification accuracy, ability to reproduce extracted prototypes, or errors on individual patterns.

\section{DISCUSSION}

The analysis of pattern classification accuracy as a function of modality indicated lower accuracy in the auditory modality. This finding does not allow the inference that visual pattern processing is, in general, superior to auditory pattern processing. An explanation of the present result, which is in opposition to the findings of Handel and Buffardi (1969) and Rubenstein and Gruenberg (1971), may lie in the rate of presentation of the auditory patterns. Preusser (1972) and Garner and Gottwald (1968) suggest that when binary auditory patterns are presented at rates of less than 2 elements/sec, as in the present study, Ss appear to process the patterns in an element-by-element fashion. This, in turn, suggests that there might well be an aspect of absolute identification of pattern elements in the present study, with the processing of auditory elements of individual pitches being more difficult than the processing of visual elements of locations in a two-dimensional spatial array. Pollack (1952) reported a maximum information transmission of approximately 2.3 bits for the absolute identification of auditory pitch, while Klemmer and Frick (1953) reported a maximum transmission of approximately 4.4 bits for the position of dots in a square. While Ss were not required to make absolute identifications in the present experiment, if absolute identification aided classification accuracy, then the superiority of visual classification is as would be expected.

The choice of translating underlying sequences to patterns of pitch auditorily and to polygons visually was completely arbitrary. It is clearly possible that there would be presentation rates and pattern formats for which equivalent performance would be found in both modalities. The problem of which modality was superior in pattern processing would dissolve, leaving the more interesting question of detailed similarities and differences between visual and auditory processing.

Auditory performance improved following performance in the visual modality, although the analogous improvement was not noted for visual processing following the auditory task. The improvement in auditory performance may best be interpreted in terms of the Ss' learning a general strategy for task performance. Ss appeared to understand the demands of the auditory task better if they had performed the visual task first. If practice in visual pattern classification led to higher accuracy in auditory classifications, then the question may be raised as to why the converse was not also true. Perhaps the simplest explanation is that the visual task was quite easy for $\mathrm{Ss}$, even without previous practice in a similar task. Performance in Group V1-A2 for visual classification was quite accurate. The visual task may well have been sufficiently easy so that Ss did not require practice in classification and so that, for some Ss, somewhat of a ceiling effect was operating in the visual modality.

The most interesting aspect of comparison between modalities is not level of accuracy but is, rather, whether there are similar processing strategies across modalities. Two results suggest that in the present experiment classification strategy was similar across modalities. First is that only the accuracy of drawing of Prototype A correlated with classification accuracy in both modalities. Ss were all trained on only Prototype A patterns. Although they were instructed that there were two classes of patterns, they could have handled the problem by testing only whether a pattern sounded like an $A$ pattern and then deciding " $A$ " vs "non-A," rather than "A" vs another distinct class, "B." If Ss were using the "A" vs "non-A" strategy, then only the extent to which they had accurately learned the $A$ prototype should correlate with their performance.

The second source of support for similarity of strategy arises from the similarity of prediction equations based on $\mathrm{DP}_{\text {own }}$ and $\mathrm{DP}_{\text {other }}$ across modalities. That the prediction equations and level of prediction accuracy were highly similar across modalities suggests that $S$ groups were relying on pattern class structure in a consistent fashion and to the same degree across modalities. It was the underlying class structure and the relationships of individual patterns to the class centroids that constituted the equivalent stimulation across modalities in the present investigation. And it was this underlying structure, measured by the variables $\mathrm{DP}_{\text {ow }}$ and DP other, to which Ss apparently responded across modalities. The greater weight assigned to $D P_{\text {ow }}$ in all regression equations can be integrated with the " $A$ " vs "non- $A$ " strategy suggested by the accuracy of prototype drawing data. A pattern from Class $A$ with a small $D P_{\text {own }}$ is clearly an $A$ pattern, and a pattern from Class $B$ with a small $D P_{\text {own }}$ is clearly not an A pattern. Thus, a small value of $D P_{\text {wn }}$ for any pattern from either class should render it easy to classify. For DP ther to be predictive requires that the $S$ be aware of two classes of patterns, rather than one Class $A$ and a series of unclassified patterns. It is of interest in this regard 
that, both for the visual and auditory modalities, $D P_{\text {other }}$ received greater weight in the regression equation for the second modality of presentation, i.e., for $\mathrm{Visual}_{2}$ over Visual, and for Auditory $_{2}$ over Auditory . This $^{\text {. Tud }}$ suggests that Ss were becoming more aware of there being two distinct classes of patterns with increased experience in pattern classification. It is possible that they were passing from an "A" vs "non- $A$ " strategy to an " $A$ " vs " $B$ " strategy as the experiment progressed.

When group data alone were considered in the analysis of classification of individual patterns, pattern processing strategy appeared similar across modalities. However, the analysis of the performance of individual Ss suggested, in contrast, no consistency across modalities. Some part of this lack of consistency might be explained by the operation of ceiling effects, particularly in the visual part of the A1-V2 condition. However, the lack of consistency was evidenced for all $40 \mathrm{Ss}$ in the error analysis, though ceiling effects were not operating for all $\mathrm{Ss}$, based on the accuracy data of Table 1. It may well be the case that intermodal pattern equivalence is a phenomenon of the "average observer," but that for any individual observer, intermodal equivalence is not at all the case. A second alternative is that for individual observers there are particular pattern input formats for which high intermodal equivalence of processing may be observed. Which of the two alternatives is true is at present unclear. Yet, the possibility that our evidence of intermodal congruence of pattern processing is the case for groups of Ss but for no individual observer is as unsettling for a general theory of pattern conception, as are average learning curves for theories of learning.

\section{REFERENCES}

AIKEN, L. S. Simultaneous processing of typal and dimensional variation among multidimensional events. Multivariate Behavioral Research, 1972, 7, 305-316.

AIKEN, L. S., \& BROWN, D. R. A feature utilization analysis of the perception of pattem class structure. Perception \& Psychophysics, 1971, 9 , 279-283.

AIKEN, L. S., \& WILliAMS, T. M. A developmental study of schematic concept formation. Developmental Psychology, 1972, in press.

BERSTED, c. T., BROWN, B. R., \& EV ANS, S. H. Free sorting with stimuli clustered in a multidimensional attribute space. Perception \& Psychophysics, 1969 , 6, 409-413

BROWN, D. R., OWEN, D. H. The metrics of visual form: Methodological dyspepsia. Psychological Bulletin, 1967, 68, 243-259.

EVANS, $S$. H. Redundancy as a variable in pattern perception. Psychological Bulletin, 1967, 67, 104-i13.

EVANS, S. H., \& ARNOULT, M. D Schematic concept formation: Demonstration in a free sorting task. Psychonomic Science, 1967, 9, 221-222.

EVANS, S. H., \& MUELLER, M. R. Vargus 9: Computed stimuli for schema research. Psychonomic Science, 1966, 6 , $511-512$.

GARNER, W. R. Uncertainty and structure as psychological concepts. New Yok: Wiley, 1962.

GARNER, W. R., \& GOTTWALD, R. L The perception and learning of temporal patterns. Quarterly Journal of Experimental Psychology, 1968, 20, 97-109.

GIBSON, J. J. Observations on active touch. Psychological Review, 1962, 69, 477-491.

HANDEL, S., \& BUFFARDI, L. Pattern perceptions integrating information presented in two modalities. Science, 1968, 162, 1026-1028.

KLEMMER, E, T., \& FRICK, F. C Assimilation of information from dot and matrix patterns. Journal of Experimental Psychology, 1953, 45, 15-19.

PETERSON, C. R., \& BEACH, L.P. Man as an intuitive statistician. Psychological Bulletin, 1967, 68, 29-46.

POLLACK, I. The information of elementary auditory displays. Journal of the Acoustical Society of America, 1952, 24, 745-749.
PREUSSER, D. The effect of structure and rate on the recognition and description of auditory temporal patterns. Perception \& Psychophysics, 1972, 11, 233-240.

RUBENSTEIN, L., \& GRUENBERG, E. M. Intramodal and crossmodal sensory transfer of visual and auditory temporal patterns. Perception \& Psychophysics, $1971,9,385-390$.

STRAHAN, R. F. A coefficient of directional correlation for time series analysis. Psychological Bulletin, 1971, 76, 211-214.

\section{NOTE}

1. The time series correlation does not take into account the size of intervals between adjacent elements, but only counts the number of corresponding transitions that are in the same or opposite direction for two ordered series of events. This correlation was chosen over the Pearson $r$, since the use of the latter correlation would imply that absolute identification of transition size by the $S$, rather than merely transition direction, was necessary for pattern classification. Intuitively, the time series correlation seemed to measure what the Ss were aware of in the auditory patterns when they stated, "The As go up at the end and the Bs go down." An example calculation is given below.

For Prototype B, the elements are

$\left[\begin{array}{lllllllll}4 & 7 & 4 & 5 & 3 & 6 & 7 & 6\end{array}\right]$.

Then the seven transitions $\left(\mathrm{X}_{\mathrm{i}+1}-\mathrm{X}_{\mathrm{i}}\right)$ are

$$
\left[\begin{array}{lllllll}
3 & -3 & 1 & -2 & 3 & 1 & -1
\end{array}\right]
$$

For elements drawn by a $S$

$$
\left[\begin{array}{llllllll}
3 & 8 & 7 & 6 & 5 & 3 & 7 & 6
\end{array}\right] \text {, }
$$

the transitions are

$$
\left[\begin{array}{lllllll}
5 & -1 & -1 & -2 & -1 & 4 & -1
\end{array}\right] \text {. }
$$

Examining (2) and (4), the computation requires that the number of corresponding transitions with the same sign be counted. For this example, $s=5$, since the first, second, fourth, sixth, and seventh transitions have the same sign. The remaining two transitions have different signs, i.e., $d=2$. Then $r=(s-d) / n=$ $(5-2) / 7=.43$

(Received for publication April 4, 1972, revision received August $21,1972$. 